\title{
Magnetic flux penetration and motion in antiferromagnetic superconductors
}

\author{
Tomasz Krzysztoń \\ Institute of Low Temperature and Structure Research, \\ Polish Academy of Sciences, \\ 50-950 Wroctaw, P.O.Box 1410, \\ Poland
}

\begin{abstract}
The paper reviews a concept of induced spin-flop domain inside vortices in an antiferromagnetic superconductor. Such phenomenon may occur when an external magnetic field is strong enough to flip over magnetic moments in the core of the vortex from their ground state configuration. The formation of the domain structure inside vortices modifies the surface energy barrier of the superconductor. During this process the entrance of the flux is stopped and a newly created state exhibits perfect shielding. Such behavior should be visible as a plateau on the dependence of flux density as a function of the external magnetic field. The end of the plateau determines the critical field, which has been called the second critical field for flux penetration. Moreover, it is predicted and described how this phenomenon modifies flux creep in layered superconductors. The various scenarios of changing the creep regime from thermal to quantum and vice versa at constant temperature are discussed. PACS numbers: 74.60.-w, 74.25.Ha.
\end{abstract}

\section{INTRODUCTION}

The discoveries of ternary Rare Earth (RE) Chevrel Phases $\mathrm{REMo}_{6} \mathrm{~S}_{8}$ and $\mathrm{RERh}_{4} \mathrm{~B}_{4} \mathrm{l}$ compounds with regular distribution of localized magnetic moments of $\mathrm{RE}$ atoms have proved conclusively the coexistence of various types of magnetism with superconductivity. Intensive experimental and theoretical works have shown that $4 \mathrm{f}$ electrons of $\mathrm{RE}$ atoms responsible for magnetism and $4 \mathrm{~d}$ electrons of molybdenum chalcogenide or rhodium boride clusters responsible for superconductivity are spatially separated and there-

fore their interaction is weak. In many of these systems superconductivity 


\section{T. Krzyszton}

coexists rather easily with antiferromagnetic order, where usually the Neel temperature $T_{N}$ is lower than the critical temperature for superconductivity $T_{c}$. On the other hand, ferromagnetism and superconductivity cannot coexist in bulk samples with realistic parameters. Quite often the ferromagnetic order is transformed into a spiral or domain-like structure, depending on the type and strength of magnetic anisotropy in the system. decades the problem of the interaction between magnetism and superconductivity has been overshadowed by high temperature superconductivity (HTS) found in copper oxides. However, the recent discovery of the presence of magnetic order in Ru-based superconductors 6 , 6 has triggered a new series of experiments and inspired a return to the so-called coexistence phenomenon. 8 Most recently, the interplay between magnetism and superconductivity was studied in d-electron $\mathrm{UGe}_{2} \mathrm{~g}$ and $\mathrm{ZrZn}_{2} 10$, where itinerant ferromagnetism may coexist with superconductivity, and in heavy fermion $\mathrm{UPd}_{2} \mathrm{Al}_{3}, 11$ where magnetic excitons are present in the superconducting phase. Among classic magnetic superconductors, the Chevrel phases have been studied most intensively. These compounds are mainly polycrystalline materials. However, some specific features can be measured only on single crystals. One such effect is a two-step flux penetration process, predicted in Ref.(12,13) and later observed solely in the antiferromagnetic superconductor (bct) $\operatorname{ErRh}_{4} \mathrm{~B}_{4} .14$ This very interesting phenomenon was recently rediscovered in $\mathrm{DyMo}_{6} \mathrm{~S}_{8}, 17$ although good quality single crystals of the classic antiferromagnetic superconductors have been a long time available and measured.

The DyMo $\mathrm{S}_{8}$ compound with $T_{c}=1.6 \mathrm{~K}$ exhibits transition from the paramagnetic to the antiferromagnetic state at $T_{N}=0.4 \mathrm{~K}$. Its crystal structure can be described as interconnected $\mathrm{Mo}_{6} \mathrm{~S}_{8}$ units and Dy ions. One such unit is a slightly deformed cube where $\mathrm{S}$ atoms sit at the corners and Mo atoms are situated at the cube-faces. The $\mathrm{Mo}_{6} \mathrm{~S}_{8}$ units are arranged in a simple rhombohedral lattice and Dy ions are located in the center of the unit cell. The magnetic moments of Dy ions form a simple structure consisting of (100) planes with moments of $8.7 \mu_{B}$ alternately parallel and antiparallel to the [111] rhombohedral axis. Neutron experiments performed on $\mathrm{DyMo}_{6} \mathrm{~S}_{8}$ in an applied magnetic field at $T=0.2 \mathrm{~K}$ have revealed in the intensity spectrum a number of peaks characteristic for ferromagnetic order. 18 These peaks begin to develop at $H_{0}=200 \mathrm{Oe}$, much below the superconducting upper critical field $H_{c 2}$. Thus, in $\mathrm{DyMo}_{6} \mathrm{~S}_{8}$ a kind of ferromagnetic order coexists with superconductivity in the same manner as antiferromagnetism. For a field applied parallel to the [111] direction (magnetic easy-axis direction), the ferromagnetic order is a spin-flop type.19

This feature is easy to understand. Consider the well known phase diagram of a two-sublattice antiferromagnet. An infinitesimal magnetic field 


\section{Antiferromagnetic superconductors}
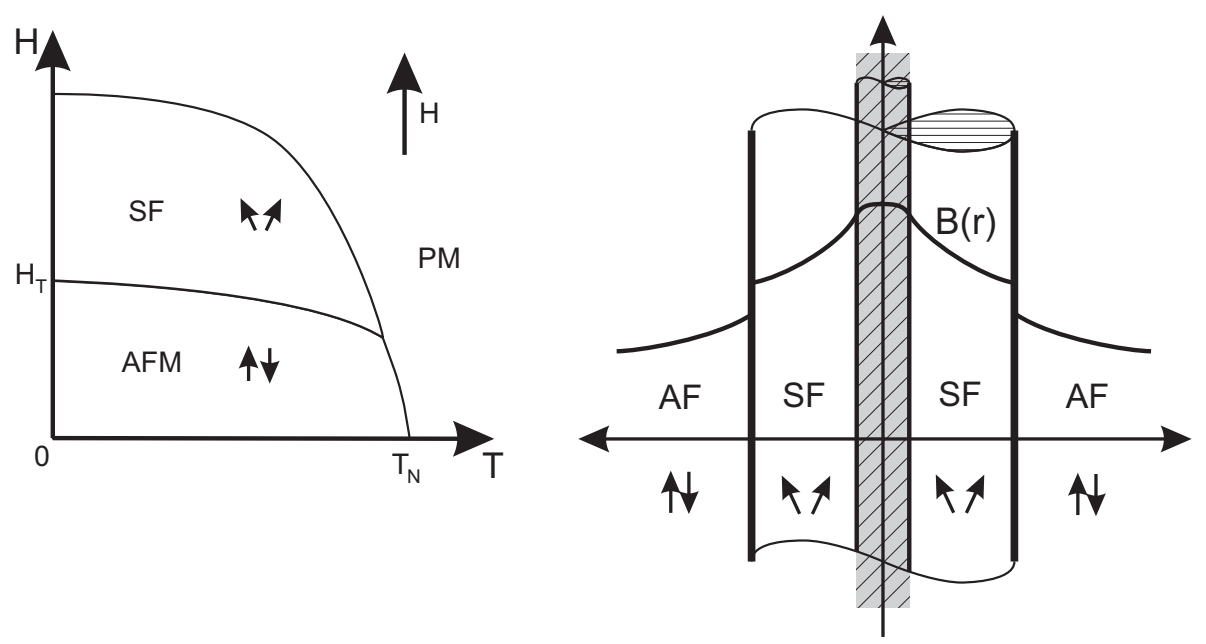

Fig. 1. On the left panel: phase diagram of two-sublattices uniaxial antiferromagnet. The external field is directed along the anisotropy axis. On the right panel: the magnetic structure of an Abrikosov vortex. Gray area corresponds to the vortex core where the spin-flop transition originates. This model has also been used by other authors 15.16 .

applied perpendicular to the easy axis makes the ground antiferromagnetic configuration unstable against the phase transformation to the canted phase. On the contrary, if the magnetic field is applied parallel to the easy axis the antiferromagnetic (AF) phase is stable up to the thermodynamic critical field $H_{T}$ as is seen on Fig.1 (left panel). When the field is further increased, a spin-flop (SF) phase develops in the system. Let us assume that in the antiferromagnetic superconductor the lower critical field fulfills the relation $H_{c 1}<\frac{1}{2} H_{T}$ and that the external field $H_{0}$ is applied parallel to the easy axis. When $H_{c 1}<H_{0}<\frac{1}{2} H_{T}$ the Abrikosov vortices appear entirely in the AF phase. When $H_{0}$ is increased beyond $\frac{1}{2} H_{T}$ the phase transition to the SF phase originates in the core because near $H_{c 1}$ the field intensity in the core is approximately twice $H_{c 1} .20$ The spatial distribution of the field across the vortex is a function decreasing from the center as is shown in Fig. 1 (right panel). Thus, the magnetic field intensity outside the core is less then $H_{T}$ and, therefore, the rest of the vortex remains in the AF phase. The radius of a spin-flop domain grows as the field is increased. The formation of domains inside the vortices should be accompanied by the modification of the surface energy barrier.13,21 This process leads to a state of the superconductor in which flux entrance is temporarily prohibited, flux density in the sample is constant as the applied field increases (see Fig. 2). In order to kill this state the external field should be increased above certain second critical field for 


\section{T. Krzysztoń}

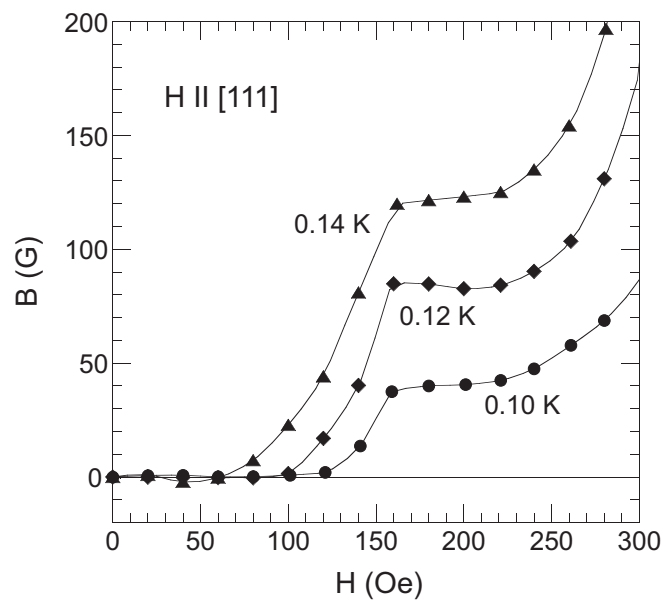

Fig. 2. Magnetic induction for $\mathrm{DyMo}_{6} \mathrm{~S}_{8}$ single crystal in the virgin state measured as a function of an applied field for three temperatures below $T_{N}=0.4 \mathrm{~K}$. The field direction is oriented parallel to the magnetic easy axis of the crystal. Each $B(H)$ curve exhibits characteristic plateau indicating that a number of vortices is kept constant when the external field is increased. The calculated values of the second critical field for flux penetration $H_{e n 2}(B)$ are in very good agreement with the measured ones.21

flux penetration $H_{e n 2}(B)$. Then, the vortices penetrating the sample will have the spin flop domains created along the cores.

The above considerations apply to the classical superconducting Chevrel phases as well as to the high $T_{c}$ superconductors. The present work is inspired by the above described discovery and the hope that the same behavior could possibly be observed in some of the layered superconducting structures. Indeed, the situation seems to be very similar in layered HTS. Here magnetic order is produced by the regular lattice of $\mathrm{RE}$ ions occupying isolating layers electrically isolated from the superconducting $\mathrm{Cu}-\mathrm{O}$ planes. Therefore spin interaction between the local magnetic moments and the conduction electrons is to weak to inhibit superconductivity. The typical example of the layered system is $\mathrm{ErBa}_{2} \mathrm{Cu}_{3} \mathrm{O}_{7}$. This compound has tetragonal unit cell with small orthorhombic distortion in the a-b plane.22.23 The $E r$ ions form two sublattice antiferromagnetic structure of magnetic moments lying parallel and antiparallel to the $b$ direction in the $a b$ plane 24 Recently discovered RE nickel boride-carbides 25 may serve as an another example of layered magnetic superconductors. The layered structure of RE nickel boride-carbides is reminiscent of that of HTS and consists of RE-carbon layers separated by $\mathrm{Ni}_{2} \mathrm{~B}_{2}$ sheets. 26,2728 29 For example in $\mathrm{ErNi}_{2} \mathrm{~B}_{2} \mathrm{C}$ the antiferromagnetic structure is associated with magnetic moments of $\mathrm{Er}^{+3}$ ions, which order be- 


\section{Antiferromagnetic superconductors}

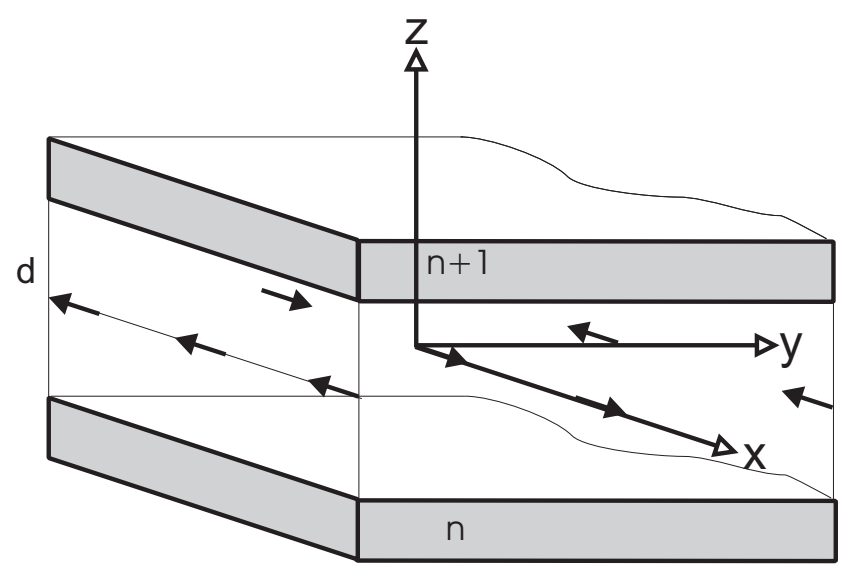

Fig. 3. Schematic drawing of a piece of the layered superconductor. The shaded areas $(n, n+1)$ represent superconducting layers. The bold arrows represent magnetic moments of $\mathrm{RE}$ ions lying in the isolating layers. The axes of the reference frame are shown.

low $6 \mathrm{~K}$ in a transversely polarized planar sinusoidal structure propagating along $a$ or $b$ axis with Er moments parallel to the $a$ or $b$ axis respectively.

\section{LONDON THEORY}

In the following we consider the structure shown on Fig. 3 that we believe simulates a real structure of many antiferromagnetic layered superconductors. A good candidate to show the above described phenomenon should possess the isolating layers with the magnetic moments of $\mathrm{RE}$ ions running parallel and antiparallel to the direction (easy axis) lying in the $a b$ plane.

We start description of our problem in terms of the Lawrence-Doniach energy functional. In this approach a layered superconductor is described by the superconducting planes with the interlayer distance d, as shown on Fig. 3. The antiferromagnetic subsystem consisting of RE ions is confined to the isolating layers. The magnetic moments are running parallel and antiparallel to the x-axis (easy axis). The Lawrence-Doniach functional is obtained from the standard Ginzburg-Landau energy by discretization of the kinetic energy in the z-direction.

$$
F_{S}=\int d^{2} r d \sum_{n}\left[\frac{\hbar^{2}}{2 m}\left|\left(-i \nabla_{(\mathbf{2})}+\frac{2 i e}{\hbar} \mathbf{A}_{(\mathbf{2})}\right) \Psi_{n}\right|^{2}+\mathrm{a}\left|\Psi_{n}\right|^{2}+\frac{1}{2} \mathrm{~b}\left|\Psi_{n}\right|^{4}+\right.
$$




\section{T. Krzysztoń}

$$
\left.+\frac{\hbar^{2}}{2 \mathcal{M} d^{2}}\left|\Psi_{n+1} \exp \left(\frac{2 e i}{\hbar} \int_{n d}^{(n+1) d} A_{z} d z\right)-\Psi_{n}\right|^{2}\right]
$$

The quantity $e, m$, denote the charge of the free electron and the mass of the current carrier in the $a b$ plane, whereas $\mathcal{M}$ denotes the mass of the current carrier in the z-direction; $\mathbf{A}=\left(A_{(2)}, A_{z}\right)$ denotes the vector potential. The antiferromagnetic two sublattices subsystem with single ion anisotropy is described with the following energy density functional

$$
f_{M}=\sum_{n}\left\{J \mathbf{M}_{1 n} \cdot \mathbf{M}_{2 n}+K \sum_{i=1}^{2}\left(M_{i n}^{x}\right)^{2}-|\gamma| \sum_{i=1}^{2} \sum_{j=x, y, z}\left(\nabla M_{i n}^{j}\right)^{2}\right\}
$$

where $\mathbf{M}_{n}=\mathbf{M}_{1 n}+\mathbf{M}_{2 n}$ is the sum of the magnetization vectors of the sublattices in the n-th insulating layer, $M_{i n}^{x}$ is the component of the magnetization sublattice vector along the anisotropy axis in the n-th layer, $J$ denotes the exchange constant between two sublattices, $K$ is the single ion anisotropy constant, $\sqrt{|\gamma|}$ is the magnetic stiffness length, and $M_{0}=\left|\mathbf{M}_{1 n}\right|=\left|\mathbf{M}_{2 n}\right|$. The components of the total magnetization vector $\mathbf{M}$ have the following form in both sublattices: $M_{i y}=M_{0} \sin \theta_{i}, M_{i x}=M_{0} \cos \theta_{i}$, where $\theta_{i}$ (canted spin angle) is the angle between the magnetization in the sublattice and the external magnetic field directed along the $x$-axis. The $\operatorname{AF}\left(\theta_{1}=0, \theta_{2}=\pi\right)$ and $\mathrm{SF}$ phases $\left(\theta_{1}=-\theta_{2}=\theta\right)$ are in thermodynamic equilibrium in an applied field equal to the thermodynamic critical field

$$
H_{T}=M_{0}[K(J-K)]^{1 / 2} .
$$

The canted spin angle of the SF phase is then expressed as

$$
\cos \theta=\frac{K M_{0}}{H_{T}}
$$

Finally we add the magnetic field energy to obtain the free energy of the entire system

$$
F=F_{s}+\int\left\{f_{M}+\frac{\mu_{0}}{2}(\mathbf{b}-\mathbf{M})^{2}\right\} d V .
$$

According to experiments the antiferromagnetic order is very weak affected by the presence of superconductivity, then it is reasonable to neglect the effect of superconductivity on the exchange interaction in F. Instead we introduce electromagnetic coupling between the magnetic subsystem and superconducting current $\mathbf{j}_{s}$. This means that both order parameters $\Psi_{n}$ and $\mathbf{M}$ are coupled through the vector potential $\mathbf{A}$

$$
\mathbf{b}=\operatorname{rot} \mathbf{A}
$$




\section{Antiferromagnetic superconductors}

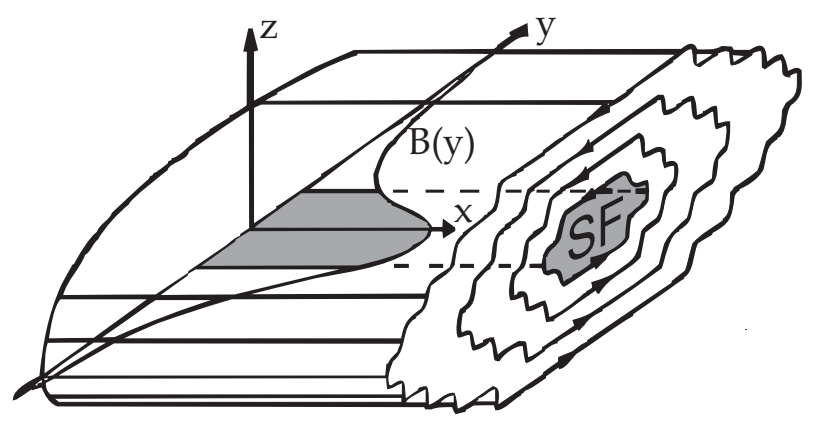

Fig. 4. Single Josephson vortex lying in the $a b$ plane along the $\hat{x}$-axis. The $\mathrm{SF}$ domain induced along the phase core is shown in the gray area.

$$
\mu_{0} \mathbf{j}_{s}=\operatorname{rot}(\mathbf{b}-\mathbf{M})
$$

where $\mathbf{b}$ is the vector of the microscopic magnetic field.

The functional (5) can be treated in the London approximation by assuming a constant modulus $\Psi_{n}$ within the planes and allowing only for the phase degree of freedom. The equilibrium conditions of the system

are the result of minimization of the Gibbs free energy functional $G=$ $F-\int \mathbf{b}(\mathbf{b}-\mathbf{M}) d V$. The London equations resulting from (司) are following:

$$
\begin{aligned}
& b_{x}+\lambda_{c}^{2} \frac{\partial}{\partial y} \operatorname{rot}_{z}(\mathbf{b}-\mathbf{M})-\lambda_{a b}^{2} \frac{\partial}{\partial z} \operatorname{rot}_{y}(\mathbf{b}-\mathbf{M})=\varphi_{0} \delta(y) \delta(z) \\
& b_{y}+\lambda_{a b}^{2} \frac{\partial}{\partial z} \operatorname{rot}_{x}(\mathbf{b}-\mathbf{M})-\lambda_{c}^{2} \frac{\partial}{\partial x} \operatorname{rot}_{z}(\mathbf{b}-\mathbf{M})=0 \\
& b_{z}+\lambda_{a b}^{2} \frac{\partial}{\partial x} \operatorname{rot}_{y}(\mathbf{b}-\mathbf{M})-\lambda_{a b}^{2} \frac{\partial}{\partial y} \operatorname{rot}_{x}(\mathbf{b}-\mathbf{M})=0
\end{aligned}
$$

$\lambda_{a b}=\lambda_{c} \sqrt{\mathcal{M} / m}, r_{j}=d \sqrt{\mathcal{M} / m}$. The London model of continuous superconductors may be used at length scales larger than the coherence length $\xi$, i.e. the core dimension. The structure of a vortex lying in the $a b$ plane in a layered superconductor with Josephson coupling between adjacent layers resembles the Abrikosov's one except that the order parameter does not vanish anywhere 31 Instead there exists a region, $r_{j}$ along the plane and $d$ perpendicular to it, where the Josephson current $j_{z}$ is of the order of the critical current. In this region, which plays the role of the vortex core, the London model fails. Away from the core the streamlines of the shielding supercurrents, which also represents contours of constant magnetic field, are elliptical except for the zigzags due to the intervening insulating layers (see Fig. (1).

The equations (8) should be supplemented by the appropriate set of differential equations describing the spatial distribution of magnetization. 


\section{T. Krzyszton}

Simple conjecture can make the calculations less complex and at the same time does not oversimplify the problem. We assume 30 that the magnetic moment is constant across the magnetic domain

$$
|\mathbf{M}|=\left\{\begin{array}{cll}
M & \text { if } & \rho<\rho_{m} \\
0 & \text { if } & \rho>\rho_{m}
\end{array}\right.
$$

where $\rho_{m}$ is the dimensionless radius of the magnetic domain in the coordinate system of the elliptical cylinder $\left(x=x, y=\lambda_{c} \rho \cos \varphi, z=\lambda_{a b} \rho \sin \varphi\right)$. Then the solution of Eq. (8) in the cylindrical reference frame for a single Josephson vortex is given by the modified Bessel functions $K_{0}$ and $I_{0}$

$$
\begin{aligned}
b_{\mathrm{SF}} & =C_{1} K_{0}(\rho)+C_{2} I_{0}(\rho) \text { for } \rho_{j}<\rho \leq \rho_{m} \\
b_{\mathrm{AF}} & =C_{3} K_{0}(\rho) \text { for } \rho>\rho_{m}
\end{aligned}
$$

( $\rho_{j}$ denotes the dimensionless phase coherence length $)$ with the following boundary conditions:

$$
\begin{aligned}
b_{\mathrm{SF}}\left(\rho_{m}\right) & =\mu_{0} H_{T}+M=B_{T} \\
b_{\mathrm{AF}}\left(\rho_{m}\right) & =\mu_{0} H_{T}
\end{aligned}
$$

These conditions, together with the flux quantization condition, are used to calculate the arbitrary constants in (10).

$$
\begin{aligned}
C_{1} & =\frac{B_{T} \rho_{m} I_{1}\left(\rho_{m}\right)-\left[\mu_{0} H_{T} \rho_{m} \frac{K_{1}\left(\rho_{m}\right)}{K_{0}\left(\rho_{m}\right)}-\frac{\varphi_{0}}{2 \pi \lambda_{c} \lambda_{a b}}\right] I_{0}\left(\rho_{m}\right)}{\rho_{m} K_{1}\left(\rho_{m}\right) I_{0}\left(\rho_{m}\right)-I_{0}\left(\rho_{m}\right)+\rho_{m} K_{0}\left(\rho_{m}\right) I_{1}\left(\rho_{m}\right)} \\
C_{2} & =\frac{B_{T}\left[\rho_{m} K_{1}\left(\rho_{m}\right)-1\right]+\left[\mu_{0} H_{T} \rho_{m} \frac{K_{1}\left(\rho_{m}\right)}{K_{0}\left(\rho_{m}\right)}-\frac{\varphi_{0}}{2 \pi \lambda_{c} \lambda_{a b}}\right] K_{0}\left(\rho_{m}\right)}{\rho_{m} K_{1}\left(\rho_{m}\right) I_{0}\left(\rho_{m}\right)-I_{0}\left(\rho_{m}\right)+\rho_{m} K_{0}\left(\rho_{m}\right) I_{1}\left(\rho_{m}\right)} \\
C_{3} & =\frac{\mu_{0} H_{T}}{K_{0}\left(\rho_{m}\right)}
\end{aligned}
$$

Finally we write free energy of the isolated vortex

$$
\begin{aligned}
\varepsilon & =\frac{\lambda_{c} \lambda_{a b}}{2 \mu_{0}} \oint_{\sigma_{1}} d \boldsymbol{\sigma}\left\{\left[\mathbf{b}_{\mathrm{SF}}(\boldsymbol{r})-\mathbf{M}\right] \times \operatorname{rot} \mathbf{b}_{\mathrm{SF}}(\boldsymbol{r})\right\} \\
& +\frac{\lambda_{c} \lambda_{a b}}{2 \mu_{0}} \oint_{\sigma_{2}} d \boldsymbol{\sigma}\left\{\mathbf{b}_{\mathrm{AF}}(\boldsymbol{r}) \times \operatorname{rotb}_{\mathrm{AF}}(\boldsymbol{r})\right\}
\end{aligned}
$$

where $\boldsymbol{r}=\left(\frac{y}{\lambda_{a b}}, \frac{z}{\lambda_{c}}\right)$ is the position of the vortex line, $\sigma_{1}$ denotes the surface of the phase core, and $\sigma_{2}$ the surface of the SF domain respectively. The 


\section{Antiferromagnetic superconductors}

integrals in (13) performed as line integrals along the contours of the cross sections of the appropriate surfaces give $\varepsilon_{1}$ - the line tension of the vortex. The minimum of $\varepsilon_{1}$ with respect to $\rho_{m}$ determines

$$
\rho_{m}^{2}=\frac{5 \phi_{0}}{8 \pi \lambda_{c} \lambda_{a b} B_{T}}
$$

\subsection{Equilibrium energy of the vortex lattice}

The London equations can be rewritten for the lattice of vortices in the following way:

$$
\mathbf{B}+\operatorname{rotrot} \mathbf{B}=\frac{\phi_{0}}{\lambda_{c} \lambda_{a b}} \sum_{m} \delta\left(\boldsymbol{r}-\boldsymbol{r}_{m}\right)
$$

where $\mathbf{r}_{m}$ specify the positions of the phase cores of the vortices. The solution of Eq. (15) is then a superposition

$$
\mathbf{B}(\boldsymbol{r})=\sum_{m} \mathbf{b}_{m}\left(\boldsymbol{r}-\boldsymbol{r}_{m}\right)
$$

of the solutions $\mathbf{b}_{m}\left(\boldsymbol{r}-\boldsymbol{r}_{m}\right)$ of isolated vortices at the points $\boldsymbol{r}_{m}$. The free energy of the system can thus be written as

$$
F=\frac{\lambda_{c} \lambda_{a b}}{2 \mu_{0}} \oint_{\sigma} d \boldsymbol{\sigma}(\mathbf{B} \times \operatorname{rot} \mathbf{B})
$$

The above symbolic surface integral is taken over the surfaces of the phase cores and the surfaces of the SF domains. The energy of the Meissner state is chosen as zero of the energy scale. Again, when the surface integrals are replaced by contour ones we get line energy of the system. This, in turn, multiplied by vortex density $n$ gives $f$ the free energy density of the system. After some transformations one can derive the following formula

$$
f=n \varepsilon_{1}+n \phi_{0} H_{T}(\ln \beta)^{-1} \sum_{m} K_{0}\left(r_{m}\right) ; \beta=\sqrt{\frac{\pi \lambda_{c} \lambda_{a b} B_{T}}{\phi_{0}}}
$$

here the sum is over all vortices excluding the one in the origin, and $r_{m}$ denotes the distance of a vortex from the origin. The lattice sum may now be replaced by integral in the $y z$-plane over a smoothed vortex density, excluding the area $n^{-1}$ associated with the single flux line in the origin. The free energy density then reduces to

$$
f=n \varepsilon_{1}+B^{2}\left(\frac{H_{T}}{B_{T}}\right)\left(\frac{\beta}{\ln \beta}\right)+B \frac{H_{T}}{4 \ln \beta} \sqrt{\frac{4 \lambda_{a b}}{27 \lambda_{c}}} \ln \left(\frac{c}{\sqrt{\lambda_{c} \lambda_{a b}}}\right)
$$




\section{T. Krzysztoń}

$$
\left(\frac{c}{\sqrt{\lambda_{c} \lambda_{a b}}}\right)^{2}=\frac{1}{\beta^{2}}\left(\frac{B_{T}}{B}\right) \sqrt{\frac{4 \lambda_{a b}}{27 \lambda_{c}}}
$$

here $c=\left|\boldsymbol{c}_{1}\right|$ denotes the length of the basal vector of the nonequilateral triangular unit cell, and $2\left|\boldsymbol{c}_{2}\right|=c \sqrt{1+\tan ^{2} \alpha}$ ( $\alpha$ is the angle between both vectors), $\tan \alpha=\sqrt{\frac{3 \lambda_{c}}{\lambda_{a b}}}$. 32 To determine the equilibrium state it is necessary to minimize the Gibbs free energy density with respect to magnetic induction. The result yields an implicit equation for the constitutive relation between magnetic induction and thermodynamic magnetic field.

$$
H-\frac{\varepsilon_{1}}{\phi_{0}}=B\left(\frac{H_{T}}{B_{T}}\right)\left(\frac{2 \beta}{\ln \beta}\right)+\frac{H_{T}}{4 \ln \beta} \sqrt{\frac{4 \lambda_{a b}}{27 \lambda_{c}}} \ln \left(\frac{c}{\sqrt{\lambda_{c} \lambda_{a b}}}\right)
$$

\section{TWO STEP FLUX PENETRATION}

Consider a semi-infinite specimen in the half space $y \geq 0$, the vortex and the external magnetic field $H_{0}$ running parallel to the surface in the $x$ direction. The presence of a surface of the superconductor leads to a distortion of the field and current of any vortex located within a distance of the order of penetration depth from the surface. To fulfill the requirement that the currents cannot flow across the surface of the superconductor we need to introduce an image vortex with the vorticity opposite to the real one. Both vortices, direct and image, interact as real ones except that the interaction is attractive. In the low flux density regime Clem 33 has shown that there exist two regions: a vortex-free region of the width $y_{f f}$ near the surface of the sample, and a constant flux density region for $y>y_{f f}$. Within the vortex-free area one can introduce the locally averaged magnetic field $B_{M}$ which is a linear superposition of the Meissner screening field, the averaged direct vortices flux density exponentially decreasing towards the surface from its interior value $B$ at $y=y_{f f}$, and averaged image vortices flux density. In our problem the $x$ component of this superposition can be approximated by

$$
B_{M}=B \cosh \left(\frac{y_{f f}-y}{\lambda_{a b}}\right)
$$

The boundary condition $B_{M}(0)=\mu_{0} H_{0}$ determines the thickness of the vortex-free region

$$
y_{f f}=\lambda_{a b} \cosh ^{-1}\left(\frac{\mu_{0} H_{0}}{B}\right)
$$

We assume that the test vortex line is lying within vortex free region at a point $\boldsymbol{r}=\left(\frac{y}{\lambda_{a b}}, 0\right)$, and its image at $\boldsymbol{r}=\left(-\frac{y}{\lambda_{a b}}, 0\right)$ outside the superconductor. Now the local field of the test vortex can be understood as a 


\section{Antiferromagnetic superconductors}

superposition of the following fields

$$
\begin{aligned}
\mathbf{B}_{\mathrm{SF}} & =\mathbf{b}_{\mathrm{SF}}(\boldsymbol{r})-\mathbf{b}_{\mathrm{AF}}(2 \boldsymbol{r})+\hat{x} B_{M}\left(\boldsymbol{r}_{f f}-\boldsymbol{r}\right) \\
\mathbf{B}_{\mathrm{AF}} & =\mathbf{b}_{\mathrm{AF}}(\boldsymbol{r})-\mathbf{b}_{\mathrm{AF}}(2 \boldsymbol{r})+\hat{x} B_{M}\left(\boldsymbol{r}_{f f}-\boldsymbol{r}\right)
\end{aligned}
$$

where $\boldsymbol{r}_{f f}=\left(\frac{y_{f f}}{\lambda_{a b}}, 0\right)$, and $\hat{x}$ denotes the unit vector in the $x$ direction. Having determined the local magnetic field we can write the Gibbs free energy of the test vortex line as

$$
\begin{aligned}
G & =\frac{\lambda_{c} \lambda_{a b}}{2 \mu_{0}} \oint_{\sigma_{1}} d \boldsymbol{\sigma}\left\{\left[\mathbf{B}_{\mathrm{SF}}(\boldsymbol{r})-2 \mu_{0} \mathbf{H}_{0}-\mathbf{M}\right] \times \operatorname{rot} \mathbf{B}_{\mathrm{SF}}(\boldsymbol{r})\right\} \\
& +\frac{\lambda_{c} \lambda_{a b}}{2 \mu_{0}} \oint_{\sigma_{2}} d \boldsymbol{\sigma}\left\{\left[\mathbf{B}_{\mathrm{AF}}(\boldsymbol{r})-2 \mu_{0} \mathbf{H}_{0}\right] \times \operatorname{rot} \mathbf{B}_{\mathrm{AF}}(\boldsymbol{r})\right\} \\
& +\frac{\lambda_{c} \lambda_{a b}}{2 \mu_{0}} \oint_{\sigma_{2}} d \boldsymbol{\sigma}\left\{\hat{x} B_{M}\left(\boldsymbol{r}_{f f}-\boldsymbol{r}\right) \times \operatorname{rot} \mathbf{B}_{\mathrm{AF}}(\boldsymbol{r})\right\}
\end{aligned}
$$

After some transformations 3033 one can obtain the Gibbs free energy per unit length $\mathcal{G}$

$$
\mathcal{G}=\mathcal{G}_{1}+\mathcal{G}_{1}^{\prime}+\mathcal{G}_{\mathcal{M}}
$$

where

$$
\begin{aligned}
\mathcal{G}_{1} & =\varepsilon_{1}-\frac{\lambda_{c} \lambda_{a b} \pi}{4 \mu_{0}} D_{1} b_{\mathrm{AF}}(2 r) \\
\mathcal{G}_{1}^{\prime} & =-\frac{\lambda_{c} \lambda_{a b} \pi}{2 \mu_{0}} D_{1}\left[b_{\mathrm{AF}}\left(r_{f f}\right)-b_{\mathrm{AF}}\left(r_{f f}+r\right)\right] \\
\mathcal{G}_{\mathcal{M}} & =-\frac{\lambda_{c} \lambda_{a b} \pi}{2 \mu_{0}}\left[D_{1} \mu_{0} H_{0}-D_{2} B_{M}\left(r_{f f}-r\right)\right]
\end{aligned}
$$

and

$$
\begin{aligned}
& D_{1}=-\left.\rho_{j} \frac{d b_{\mathrm{SF}}(\rho)}{d \rho}\right|_{\rho=\rho_{j}}-\left.\rho_{m} \frac{d b_{\mathrm{SF}}(\rho)}{d \rho}\right|_{\rho=\rho_{m}}-\left.\rho_{m} \frac{d b_{\mathrm{AF}}(\rho)}{d \rho}\right|_{\rho=\rho_{m}} \\
& D_{2}=-\left.\rho_{j} \frac{d b_{\mathrm{SF}}(\rho)}{d \rho}\right|_{\rho=\rho_{j}}-\left.\rho_{m} \frac{d b_{\mathrm{SF}}(\rho)}{d \rho}\right|_{\rho=\rho_{m}}-\left.2 \rho_{m} \frac{d b_{\mathrm{AF}}(\rho)}{d \rho}\right|_{\rho=\rho_{m}}
\end{aligned}
$$

$\mathcal{G}_{1}$ describes the interaction of the test vortex with its image, $\mathcal{G}_{1}^{\prime}$ is a correction term introduced by Clem 33 and $\mathcal{G}_{\mathcal{M}}$ describes the interaction energy of the test vortex with the modified Meissner field. To find the conditions of the vortex entrance and exit, one has to solve a force balance equation for the test vortex, at the surface of the sample, and at the edge of the flux-filled area, respectively. A calculation using $\mathcal{G}_{1}$ and $\mathcal{G}_{\mathcal{M}}$ alone gives non vanishing force on the test vortex at $\boldsymbol{r}=\boldsymbol{r}_{f f}$. However, the force should be zero there, 


\section{T. Krzyszton}

because $\mathcal{G}_{\mathcal{M}}$ is supposed to account for all the image vortices. To avoid double counting the image vortex one can subtract from the self-energy a contribution of the excess image fixed at $\boldsymbol{r}=-\boldsymbol{r}_{f f}$. One can easily check that $\mathcal{G}_{1}^{\prime}$ is negligible at the surface of the sample and has no influence on the conditions of the flux entrance. When the flux starts to enter the sample, $H_{0}=H_{e n 2}(B)$,

$$
y_{f f}=y_{e n}=\lambda_{a b} \cosh ^{-1}\left(\frac{\mu_{0} H_{e n 2}(B)}{B}\right)
$$

and the energy barrier is moved toward the surface within $\rho_{m}$. Thus, one can derive from the force balance equation

$$
-\left.\frac{D_{1}}{2 D_{2}} \frac{d b_{\mathrm{AF}}(\rho)}{d \rho}\right|_{\rho=\rho_{m}}=B \sinh \left(\frac{y_{e n}}{\lambda_{a b}}\right)
$$

The left hand side of the above equation gives $H_{e n 2}(0)=H_{T} \beta(2 \ln \beta)^{-1}$. This field may be thought as the second critical field for flux penetration calculated in the single vortex approximation 30 . Combining Eqs. (27) and (28) one can finally obtain

$$
H_{e n 2}(B)=\sqrt{B^{2}+\left(\frac{\mu_{0} H_{T} \beta}{2 \ln \beta}\right)^{2}}
$$

In the opposite case, when the flux exits the sample, the surface energy barrier tends to the edge of the flux-filled zone. Similar considerations as the above show that

$$
\mu_{0} H_{e x 2}(B) \simeq B
$$

The measure of the height of the energy barrier against flux entrance is

$$
\Delta H_{e n}(B)=\left|H_{e n 2}(B)-H_{e q}(B)\right|,
$$

and against flux exit

$$
\Delta H_{e x}(B)=\left|H_{e q}(B)-H_{e x 2}(B)\right|,
$$

where $H_{e q}$ is given by Eq. (19).

Let us make a short summary of the calculations and visualize the results on schematic magnetization curve shown in the Fig. 5. When the external field is not strong enough to create the SF domains inside vortices, than the magnetization process of the sample being entirely in the AF phase is as follows. The vortices without magnetic structure start to enter the specimen at $H_{e n 1}$. When the field is increased up to the value $H_{p l}$, which is of the order of $H_{T}$, the SF domains are created. Now, the screening current must 


\section{Antiferromagnetic superconductors}

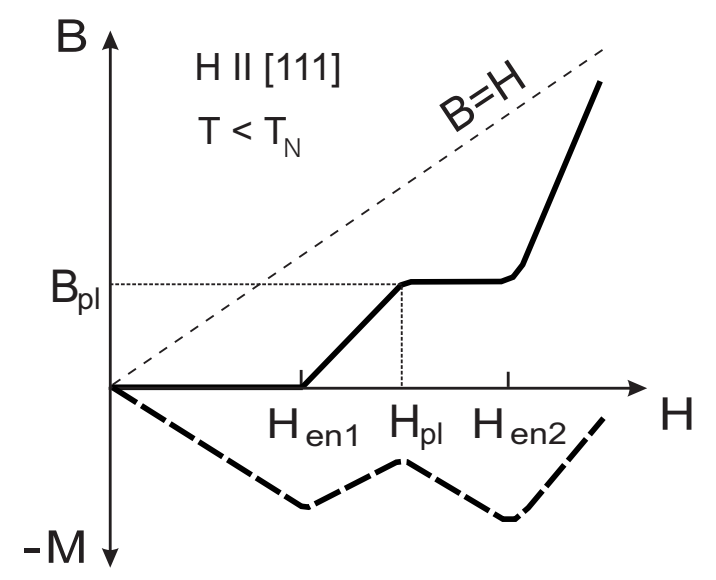

Fig. 5. Schematic drawing of the magnetization process. $H_{e n 1}$ denotes the first penetration field for vortices without magnetic structure. $H_{p l}$ is the applied field which originates SF transitions inside vortices, and $B_{p l}$ is the corresponding flux density. $H_{e n 2}$ is the entrance field for the vortices possessing magnetic structure. The lower dashed line shows $M(H)$ dependence. The region of plateau on $B(H)$ corresponds to the region of the second negative slope on $M(H)$.

redistribute its flow in order to keep constant the flux carried by the vortex. This feature is easily seen from Eqs. (10)-(12). The redistribution of the screening current changes the surface energy barrier preventing vortices from entering the sample as expressed in (28). It means that the density of vortices $n$ is kept constant. Consequently the averaged flux density in the sample $B=n \varphi_{0}$ remains constant when the external field is increased. In Fig. 5 this feature is visible as a plateau on the $B(H)$ curve, or alternatively as a second negative slope on the $M(H)$ curve. The vortices start to penetrate the sample when the external field reaches the right edge of the plateau. We call this value, given by (29), second critical field for flux penetration $H_{e n 2}$.

To find the thermodynamic critical field $H_{T}$, and then to calculate $H_{e n 2}(B)$ the following argumentation is used. At low fields, in the vicinity of the lower critical field $H_{c 1}$, the intensity of the field in the vortex core is $2 H_{c 1} .31$ When the external field is increased the field intensity in the vortex core increases because of the superposition of the fields of the surrounding vortices. The field intensity in the core must reach $H_{T}$ in order to originate a transition to the SF phase. Thus, taking into account only the nearest neighbors we can write for the nonunilateral triangular lattice

$$
H_{T}=2 H_{c 1}+z \frac{\varphi_{0}}{\pi \lambda_{c} \lambda_{a b} \mu_{0}}\left[K_{0}\left(\frac{c}{\lambda_{a b}}\right)+2 K_{0}\left(\frac{c}{2 \lambda_{a b}} \sqrt{\frac{3 \lambda_{c}}{\lambda_{a b}}}\right)\right]
$$




\section{T. Krzysztoń}

here $c$ corresponds to the value $B_{p l}$ of the flux density for which the penetration process stops, see Fig. 5. From the relation $B_{p l}=2 \varphi_{o} \sqrt{\lambda_{a b}} /\left(c^{2} \sqrt{3 \lambda_{c}}\right)$ one can compute $c$, which in turn may be inserted back into Eq. (31). It is easy to estimate the saturation magnetization $M_{0}$ taking into account the volume of the elementary cell. Then, equations (3) and (蛋) can be used to calculate $M$ in the SF-phase domain

$$
M=2 M_{0} \cos \theta=\frac{2 K M_{0}^{2}}{H_{T}}
$$

\section{INTRINSIC PINNING}

The first quantitative approach toward intrinsic pinning in layered superconductors was based on the observation that the superconducting order parameter should have a periodic spatial variation across the layers. For the present considerations, however, the method of critical nucleus developed in 34 is much more convenient. The activated nucleus consists of a kinkantikink excitation, that is, a vortex line segment is thrown to the adjacent layer, thereby creating two pancake vortices of opposite vorticity, as shown in the Fig. 6. The activation energy can be regarded as the energy barrier for

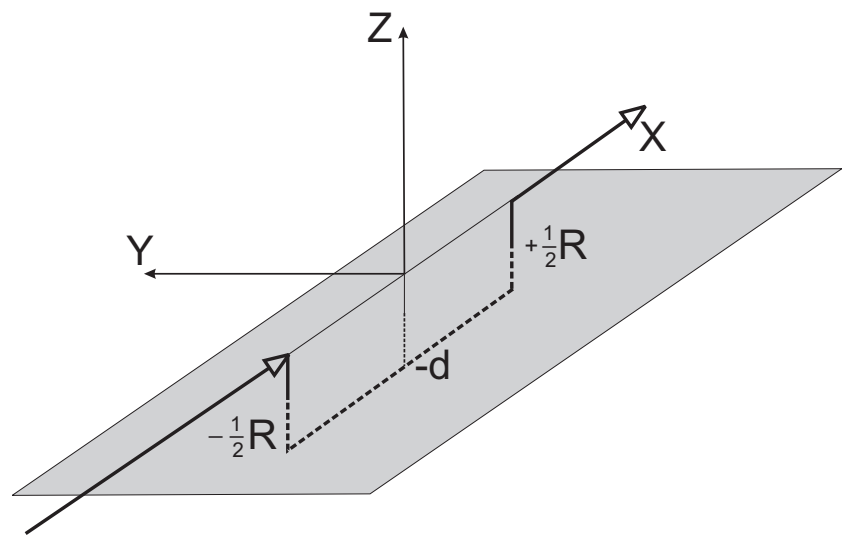

Fig. 6. The double-kink excitation form a critical nucleus. The part of the vortex is thrown to the adjacent layer.

intrinsic pinning. Depending on the magnitude of the driving current density the process may continue as the single vortex activation or the activation of the vortex bundle. 


\section{Antiferromagnetic superconductors}

\subsection{Thermal activation of vortices}

First, consider the activation of a segment of a vortex to the neighboring interlayer spacing (see Fig. 6). The energy associated with this process can be written as:

$$
U=\delta E+V_{K,-K}(R)-\left(j-j_{0}\right) \varphi_{0} d R
$$

The subscript "a" or "b" of $U, \delta E$ and $j_{0}$ indicates that this quantity is calculated for $a$ or $b$ direction in the plane. $\delta E$ is the amount of condensation and magnetic domain energy that is lost at two points of the layer threaded by the kinks separated by a distance $R . \quad V_{K,-K}(R)$ is the kink-antikink interaction energy. The term proportional to the driving current $j$ is due to the Lorentz force. The term proportional to $j_{0}$ is the energy associated with the distortion of the line due to the formation of nucleus. This term can be estimated from the simple considerations

$$
j_{0} \varphi_{0} d \sim \frac{1}{2} \int d y d z C(y, z)\left(\frac{\partial u_{z}}{\partial z}\right)^{2}
$$

where the Fourier transform of the compression modulus is given by 35

$$
C\left(k_{y}, k_{z}\right)=\frac{B^{2}}{\mu_{0}\left(1+\lambda_{a b}^{2} k_{z}^{2}+\lambda_{c}^{2} k_{y}^{2}\right)}
$$

By taking $d y d z \sim \varphi_{0} / B, u_{z} \sim d, \frac{\partial}{\partial z} \sim k_{z} \sim k_{y}\left(\lambda_{c} / \lambda_{a b}\right)$ the integral can be estimated as follows

$$
j_{0 b}=\frac{B d}{4 \lambda_{a b}^{2}} .
$$

However, in the $a$ direction, according to (14) we have an additional contribution from the magnetic domain $d y d z \sim 5 \varphi_{0} / 8 B_{T}$. Thus we get

$$
j_{0 a}=j_{0 b}+\frac{5 d B_{T}}{128 \lambda_{a b}^{2}} .
$$

As the current density $j$ drops below $j_{0}$ a single-vortex line can no longer be activated due to the confinement energy provided by the vortex lattice. The current $j_{0}$ plays very important role in all above calculations. For the following values $d \sim 10^{-9} \mathrm{~m}, \xi_{c} \sim 3 \times 10^{-10} \mathrm{~m}, \xi_{a b} \sim 3 \times 10^{-9} \mathrm{~m}, r_{j} \sim 10^{-8} \mathrm{~m}$ and their typical temperature dependence we can estimate

$$
\frac{j_{0 b}}{j_{G L}} \sim \frac{B d \xi_{a b}}{\varphi_{0}} \sim 10^{-3} B \sqrt{\frac{T_{c}}{T_{c}-T}}
$$

where $j_{G L}=4 \epsilon_{0} /\left(\varphi_{0} \xi_{a b} 3 \sqrt{3}\right)$ is the Ginzburg-Landau critical current density and $\epsilon_{0}=\varphi_{0}^{2} /\left(16 \pi^{2} \mu_{0} \lambda_{a b}^{2}\right)$. Since the depairing current is of the order of 


\section{T. Krzysztoń}

$10^{13}\left[T_{c} /\left(T_{c}-T\right)\right]^{3 / 2}$ then the current $j_{0 b}$ is of the order of $10^{10} B\left[T_{c} /\left(T_{c}-T\right)\right]$. Although there are no precise measurements of SF transition in the AF HTS, we assume that $\mu_{0} H_{T} \sim 40 \mathrm{mT}$. The typical value of $5.5 \mu_{B}$ per RE atom per unit cell gives $M \sim 0.37 T$. It is possible now to estimate the change of $j_{0}$ due to the creation of SF domain along the vortex :

$$
\frac{j_{0 b}}{j_{0 a}} \sim 1+0.625 \frac{B_{T}}{B} \sim 3
$$

providing that $H_{T} / H_{c 1}-1<<1$. The energy $\delta E$ is calculated from Eq. (8) with the right-hand sides representing the vortex cores: $: 36$

$$
\left\{|x|>\frac{R}{2}, y=0, z=0\right\}\left\{x= \pm \frac{R}{2}, y=0,0<z<-d\right\}\left\{|x|<\frac{R}{2}, y=0, z=-d\right\}
$$

The solution is then substituted to the free energy functional (5). Taking the limit $R \rightarrow \infty$ we exclude the energy of the kink-antikink interaction. Because the calculations are involved we write down only the results.

$$
\begin{gathered}
\delta E_{b}=2 d \epsilon_{0} \ln \frac{r_{j}}{\xi_{a b}} \\
\delta E_{a}=d \epsilon_{a} \ln \frac{r_{j}}{\xi_{a b}}
\end{gathered}
$$

where $\epsilon_{a}=\frac{77}{64} \epsilon_{0} \ln \left[\varphi_{0} /\left(\pi r_{j}^{2} B_{T}\right)\right]$. The energy of kink-antikink interaction was calculated in 34

$$
V_{K,-K}(R)=-\frac{d^{2} \epsilon_{0}}{2 \lambda_{a b}} f\left(\frac{R}{\lambda_{c}}\right)
$$

where

$$
f\left(\frac{R}{\lambda_{c}}\right)=\left\{\begin{array}{ccc}
\left(\lambda_{c} / R\right)-\ln \left(r_{j} / \xi_{a b}\right) & \text { for } & r_{j}<<R<< \\
2\left(\lambda_{c} / R\right)^{3} \exp \left(-R / \lambda_{c}\right) & \text { for } & R>>\lambda_{c}
\end{array}\right.
$$

If we introduce the quantity $I_{a, b}=2\left(j-j_{0 a, b}\right) /\left(j_{G L} 3 \sqrt{3}\right)$, then Eq. (33) can be rewritten in the following way

$$
\begin{aligned}
U_{b} & =2 d \epsilon_{0}\left\{\ln \frac{r_{j}}{\xi_{a b}}+I_{b} \frac{R}{\xi_{a b}}-\frac{d}{4 \lambda_{a b}} f\left(\frac{R}{\lambda_{c}}\right)\right\} \\
U_{a} & =d\left\{\epsilon_{a} \ln \frac{r_{j}}{\xi_{a b}}+2 \epsilon_{0} I_{a} \frac{R}{\xi_{a b}}-\frac{d \epsilon_{0}}{2 \lambda_{a b}} f\left(\frac{R}{\lambda_{c}}\right)\right\}
\end{aligned}
$$

The critical size of the nucleus $R_{c}$ is given as a minimum of Eq. (39) with respect to $R$. In the approximation $r_{j}<<R<<\lambda_{c}$ corresponding to the current regime $\xi_{c} d / \lambda_{a b}^{2}<<I_{a, b}<<\xi_{c} / d$ we get 


\section{Antiferromagnetic superconductors}

$$
\begin{aligned}
R_{c a, b}^{2} & =\xi_{a b}^{2} \frac{d}{4 I_{a, b} \xi_{c}} \\
U_{b}^{c} & =2 d \epsilon_{0}\left\{\ln \left(\frac{r_{j}}{\xi_{a b}}\right)-\sqrt{\frac{d I_{b}}{\xi_{c}}}\right\} \\
U_{a}^{c} & =d\left\{\epsilon_{a} \ln \left(\frac{r_{j}}{\xi_{a b}}\right)-2 \epsilon_{0} \sqrt{\frac{d I_{a}}{\xi_{c}}}\right\}
\end{aligned}
$$

For the opposite case $R>>\lambda_{c}$ and $\xi_{c} d / \lambda_{a b}^{2}>>I_{a, b}$

$$
\begin{aligned}
R_{c a, b} & =\lambda_{c} \ln \left(\frac{d \xi_{c}}{I_{a, b} \lambda_{a b}^{2}}\right) \\
U_{b}^{c} & =2 d \epsilon_{0}\left\{\ln \left(\frac{r_{j}}{\xi_{a b}}\right)-\frac{\lambda_{a b} I_{b}}{\xi_{c}} \ln \left(\frac{d \xi_{c}}{I_{b} \lambda_{a b}^{2}}\right)\right\} \\
U_{a}^{c} & =d\left\{\epsilon_{a} \ln \left(\frac{r_{j}}{\xi_{a b}}\right)-\frac{\lambda_{a b} I_{a}}{\xi_{c}} \ln \left(\frac{d \xi_{c}}{I_{a} \lambda_{a b}^{2}}\right)\right\}
\end{aligned}
$$

When the driving current drops below $j_{0}$ the critical nucleus is $3 \mathrm{D}$ object. In our case it is a parallelepiped of the height $R$ along the bundle and of the section $S$ across it. The activation energy is a sum of the volume energy due to the Lorentz force and the surface energy.

$$
U_{a, b}=-j B d R S+\delta E_{a, b}\left(\frac{B S}{\varphi_{0}}\right)+j_{0 a, b} d R \sqrt{B S \varphi_{0}}
$$

The second term is the loss of condensation energy (and magnetic domain energy in the case of $a$ direction) on both surfaces perpendicular to the bundle multiplied by the number of vortices threading these surfaces. The third term is the elastic energy released in the surface parallel to the shifted vortex $j_{0 a, b} d R \varphi_{0}$ multiplied by the number of shifted vortices $\sqrt{B S / \varphi_{0}}$ (one vortex per plane). The critical nucleus is then $S_{c}=\left(\varphi_{0} / B\right)\left(j_{0 a, b} / j\right)^{2}, R_{c}=$ $\delta E_{a, b} /\left(j d \varphi_{0}\right)$, and the activation energy is

$$
U_{a, b}^{c}=\delta E_{a, b}\left(\frac{j_{0 a, b}}{j}\right)^{2} .
$$

\subsection{Thermal creep}

The resistive mechanism in the mixed state is determined by the activation process leading to magnetic flux motion (creep). This motion induces 


\section{T. Krzysztoń}

electric field which can be observed on the current-voltage characteristic. We consider the motion of activated kinks along the layers of the length $L$ along the magnetic field direction. Assume that each double-kink can reach the boundary of the sample before the new one is created. The mean electric field associated with this motion is given by

$$
E=B P L d S_{c}
$$

where $P$ is the activation probability per unit volume and unit time. For thermal activation this probability is given by $P \sim \exp \left(-U_{c} / k_{B} T\right)$. There is however a crossover temperature $T_{0}$ below which quantum tunneling of vortices is dominating. The probability for quantum tunneling is finite even for $T=0$. The Neel temperature for layered antiferromagnetic superconductors varies from hundreds of $\mathrm{mK}\left(0.6 \mathrm{~K}\right.$ for $\left.\mathrm{ErBa}_{2} \mathrm{Cu}_{3} \mathrm{O}_{7}\right)$ to several Kelvin (6.8K for $\mathrm{ErNi}_{2} \mathrm{~B}_{2} \mathrm{C}$ ) and therefore both mechanisms of activation are present in these compounds. The preexponential factors and $T_{0}$ cannot be calculated in the framework of thermodynamic considerations alone. Fortunately, it was shown in 37 that the activation probability of macroscopic quantum excitations is proportional to $j^{3}$. Thus we can assume that $P=\alpha_{0} j^{3} \exp \left(-U_{c} / k_{B} T\right)$. Now we can calculate the current-voltage characteristics for the current density $j<<j_{0}$

$$
\begin{aligned}
& E_{a}=\varphi_{0} d L \alpha_{0} j_{0 a}^{2} j \exp \left\{-\frac{\delta E_{a}}{k_{B} T}\left(\frac{j_{0 a}}{j}\right)^{2}\right\} \\
& E_{b}=\varphi_{0} d L \alpha_{0} j_{0 b}^{2} j \exp \left\{-\frac{\delta E_{b}}{k_{B} T}\left(\frac{j_{0 b}}{j}\right)^{2}\right\}
\end{aligned}
$$

This almost linear dependence of $E$ on $j$ indicates that the resistive mechanism of bundle activation follows $\mathrm{Ohm}$ law. We can also calculate the rate of flux creep due to the thermal activation of vortices. To do this consider hollow cylindrical sample of a radius $r$ and the wall thickness $l<<r$ placed in the magnetic field $B_{e x}>B_{c 1}$ applied parallel to the cylinder axis. The sample has the trapped field $B_{i n}$ inside the hole and corresponding trapped flux $\Phi=\left(B_{i n}-B_{e x}\right) \pi r^{2}$. According to the Faraday's law electric field due to the change of the trapped flux is equal to $\left(\mu_{0} / 2\right) \operatorname{lr}(d j / d t)$. Combining this result with Eq. (44) we have finally

$$
B P L d S_{c}+\frac{1}{2} \mu_{0} l r \frac{d j}{d t}=0
$$

This equation can be solved analytically only in the case of the weak currents. Consequently for excitations in the form of bundle of vortices the above 


\section{Antiferromagnetic superconductors}

equation is written as

$$
\Omega j \exp \left\{-\frac{\delta E_{a, b}}{k_{b} T}\left(\frac{j_{0 a, b}}{j}\right)^{2}\right\}+\frac{d j}{d t}=0
$$

where $\Omega=\varphi_{0} \alpha_{0} j_{0}^{2} /\left(\mu_{0} \gamma\right)$ and $\gamma=r l /(L d)$ is the factor determined by the geometry of the sample. The solution of $\mathrm{Eq}$ (47) is given in terms of exponential integrals and for the case of $j_{0 a, b} / j-1<<1$ it can be approximated as:

$$
\frac{j(0)}{j(t)}-1=\frac{\Phi(0)}{\Phi(t)}-1=\frac{k_{B} T}{2 \delta E_{a, b}}\left(\frac{j(0)}{j_{0 a, b}}\right)^{2} \ln \left(1+\omega_{a, b} t\right)
$$

where

$$
\omega_{a, b}=\frac{4 \varphi_{0} \alpha_{0} \delta E_{a, b}}{\mu_{0} \gamma}\left(\frac{j_{0 a, b}}{j(0)}\right) \exp \left\{-\frac{\delta E_{a, b}}{k_{b} T}\left(\frac{j_{0 a, b}}{j(0)}\right)^{2}\right\}
$$

This result is in agreement with the experiments on HTS. 38 For $0<<t<<$ $1 / \omega$ the change of trapped flux is linear in time and for $t>>1 / \omega$ logarithmic. In antiferromagnetic superconductors, however, we see additional change of characteristic frequency as the magnetic field changes its direction in the $a b$ plane.

\subsection{Quantum creep}

Below $T_{0}$, the quantum activation probability is essentially independent of temperature $P \sim \exp (-S / \hbar)$ and is interpreted as arising from the quantum tunneling of vortices through intrinsic pinning potential.39, 40 In the folowing we show a considerable change of tunneling rate and crossover temperature due to the SF phase transition around the vortex core. Now, consider the vortex line as a straight string-like object of an effective mass $m$ per unit length trapped into a metastable state in an intrinsic pinning potential $V(u)$ and exposed to continuous deformation $u(x, t)$ in the $\hat{z}$ direction. 11 The magnetic field is applied in $\hat{x}$ direction ( $a$ direction on Fig. 3). In the semi-classical approximation the quantum decay rate is calculated as a saddle-point solution (bounce) of the Euclidean action $S$ for the string

$$
\begin{array}{r}
S=\int_{-\infty}^{\infty} d x \int_{0}^{\hbar \beta} d \tau\left\{\frac{1}{2} m\left(\frac{\partial u}{\partial \tau}\right)^{2}+\frac{\varepsilon_{1}}{2}\left(\frac{\partial u}{\partial x}\right)^{2}+V(u)+\right. \\
\left.+\frac{\eta}{2 \pi} \int_{0}^{\hbar \beta} d \tau^{\prime}\left|\frac{u(x, \tau)-u\left(x, \tau^{\prime}\right)}{\tau-\tau^{\prime}}\right|^{2}\right\}
\end{array}
$$




\section{T. Krzyszton}

Here $\beta=\left(k_{B} T\right)^{-1}, \eta$ is the viscosity coefficient and $\tau$ denotes imaginary time. The pinning potential $V(u)$ consists of intrinsic periodic part and the Lorentz potential:

$$
V(u)=-\frac{\varphi_{0} j_{0} d}{2 \pi} \cos \left(\frac{2 \pi u}{d}\right)-\varphi_{0} j u .
$$

For large current, this potential can be expanded around the inflection point to give

$$
V(u)=V_{0}\left[\left(\frac{u}{w}\right)^{2}-\left(\frac{u}{w}\right)^{3}\right],
$$

where $V_{0}=\frac{2}{3} \frac{\varphi_{0} j_{0}^{2} \pi^{2}}{d^{2}} w^{3}$ and $w=\frac{3 d}{\pi}\left(\frac{j_{0}-j}{2 j_{0}}\right)^{\frac{1}{2}}$ may be thought as the width of the barrier because $V(0)=V(w)=0$. The last term in (49) is the socalled Caldeira-Leggett action, 12 which describes ohmic damping produced by the coupling to the heat-bath of harmonic oscillators. The line tension $\varepsilon_{1}$ is different for vortices in two different orientations in the $a b$ plane. As previously discussed the vortices lying parallel to $b$ direction and those laying in the $a$ direction but created in the magnetic field fulfilling relation $H_{c 1}<$ $H<\frac{1}{2} H_{T} 30$ have the line tension equal to

$$
\varepsilon_{b}=\epsilon_{0} \ln \frac{\lambda_{a b}}{d}
$$

For those vortices lying in the $a$ direction but possessing spin flop domain, we write the following expression 30

$$
\varepsilon_{a}=\frac{\varphi_{0} H_{T}}{2}+\frac{9}{128} \epsilon_{0} \ln \frac{\varphi_{0}}{\pi r_{j}^{2} B_{T}} .
$$

In the semiclassical approximation the decay rate is given by the value of the action on a classical trajectory obtained from the Euler-Lagrange equations of the motion

$$
-m \frac{\partial^{2} u}{\partial \tau^{2}}-\varepsilon_{1} \frac{\partial^{2} u}{\partial x^{2}}+V^{\prime}(u)+\frac{\eta}{\hbar \beta} \int_{0}^{\hbar \beta} d \tau \frac{\partial u}{\partial \tau} \cot \frac{\pi}{\hbar \beta}\left(\tau-\tau^{\prime}\right)=0
$$

The trajectory $u_{0}(x)$ for static solution of Eq. (54) gives the activation energy in the thermal regime $T>T_{0}$. Below this crossover temperature a new kind of trajectory, periodic in imaginary time, develops. Therefore, $u(x, \tau)$ can be expanded in the Fourier series with Matsubara frequencies

$$
u(x, \tau)=\sum_{n=0}^{\infty} u_{n}(x) \cos \left(\omega_{n} \tau\right) \quad ; \quad \omega_{n}=\frac{2 \pi n}{\hbar \beta} .
$$




\section{Antiferromagnetic superconductors}

Substituting this expansion into (54) and linearizing potential around the static solution $u_{0}(x)$ one obtains

$$
-\varepsilon_{1} \frac{\partial^{2} u_{n}}{\partial x^{2}}+V^{\prime \prime}\left(u_{0}\right) u_{n}=-\left(\eta \omega_{n}-m \omega_{n}^{2}\right) u_{n} .
$$

The above equation has three discrete solutions, 13 the unstable one corresponding to the tunnelling process determines the crossover temperature

$$
k_{B} T_{0}=\frac{\hbar \eta}{4 \pi m}\left\{\left[1+\frac{20 \pi \varphi_{0} j_{0} m}{d \eta^{2}}\left(\frac{j_{0}-j}{2 j_{0}}\right)^{\frac{1}{2}}\right]^{\frac{1}{2}}-1\right\}
$$

The above calculations apply to both kind of vortices. The only difference is their effective mass and viscosity coefficient. It is possible to express these parameters as the function of condensation energy accumulated in the vortex cores. For the stationary flux flow the viscous force $\eta \frac{\partial u}{\partial t}$ is equal to Lorentz force. The electric field generated by the moving vortex is $E=B \frac{\partial u}{\partial t}$, so we get $E=\frac{\varphi_{0} B}{\eta} j=\rho j=\rho_{N} \frac{B}{H_{c 2}} j$ where $\rho_{N}$ is the normal phase resistivity in the $a b$ plane and $H_{c 2}$ is the upper critical field parallel to the layers. Finally,

$$
\eta=\frac{\varphi_{0} H_{c 2}}{\rho_{N}}=\frac{\varphi_{0} \kappa H_{c} \sqrt{2}}{\rho_{N}}=\varepsilon_{1} \frac{4 \sqrt{3} \kappa^{2}}{\pi \rho_{N} \ln \kappa},
$$

where $H_{c}=\frac{\varepsilon_{l} \kappa 2 \sqrt{6}}{\pi \varphi_{0} \ln \kappa}$ is calculated from the constitutive relation $\varepsilon_{1}=H_{c 1} \varphi_{0}$. The effective mass of the vortex can be deduced from the work of Suhl.44 He derived the core contribution to the inertial mass $m_{\text {core }}=\frac{3}{8} m_{e} \frac{\xi^{2} H_{c}^{2} \mu_{0}}{\epsilon_{F}}$, where $m_{e}$ denotes the mass of the electron and $\epsilon_{F}$ is the Fermi energy, and the electromagnetic contribution coming from the energy of the electric field induced by the moving flux. Simple estimation shows that this contribution in layered superconductors is $10^{-4}$ of the core contribution. Therefore,

$$
m=\varepsilon_{1}^{2} \frac{9 \lambda_{a b}^{2} m_{e} \mu_{0}}{\varphi_{0}^{2} \pi^{2} \epsilon_{F}(\ln \kappa)^{2}} .
$$

It is possible now to relate the crossover temperature in Eq. (57) to the line tension of the vortex

$$
T_{0}=\alpha \varepsilon_{1}^{-1} .
$$

The coefficient $\alpha$ depends on the material constants and current intensity.

\subsection{Crossover from quantum to thermal creep}

As was already mentioned there are two types of vortex lines in the system. The first ones, without magnetic domain, occur when the field 


\section{T. Krzysztoń}

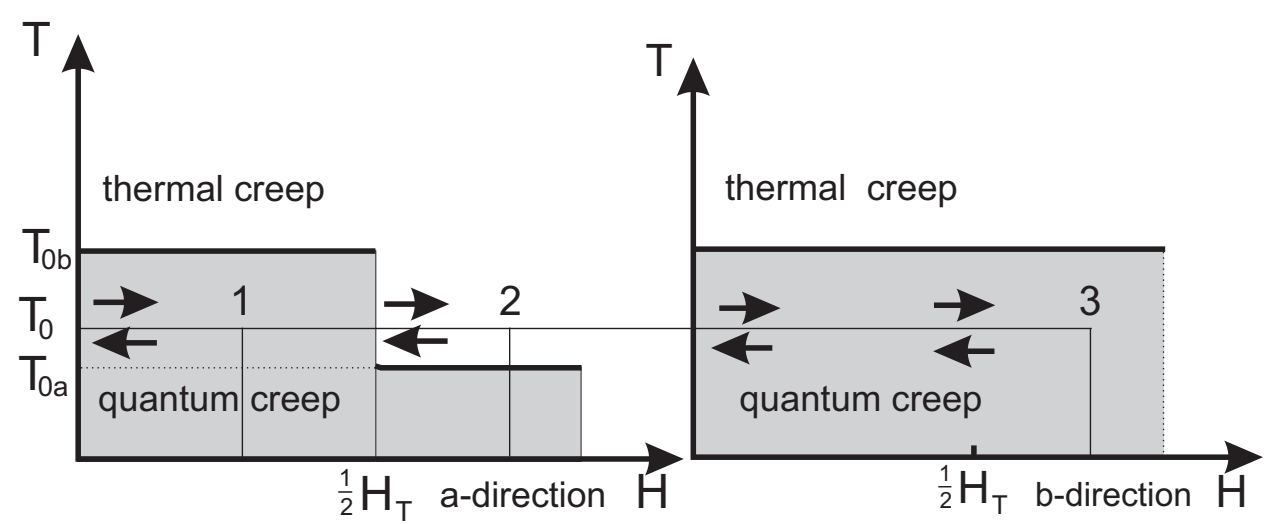

Fig. 7. Schematic diagrams showing possible ways ( marked by arrows ) of changing quantum creep behavior in the system to thermal one, and vice versa. Shaded areas on the diagrams correspond to quantum creep regime 4 .

is applied in the $a$ or $b$ direction, but its intensity does not exceed $\frac{1}{2} H_{T}$. Eq. (52) gives their line tension and the related crossover temperature $T_{0 b} \sim$ $\varepsilon_{b}^{-1}$. The other type, possessing magnetic domain, occur when the field is applied in the $a$ direction and its intensity exceeds $\frac{1}{2} H_{T}$. Eq. (53) gives their line tension and the crossover temperature as $T_{0 a} \sim \varepsilon_{a}^{-1}$. It is easy to see that $\varepsilon_{a}>\varepsilon_{b}$ and therefore $T_{0 b}>T_{0 a}$. The above calculations lead to the following conclusion. It is possible to switch the creep regime at constant temperature. To do this, one needs to change the field intensity or simply change the field direction in the $a b$ plane. The diagrams in Fig. 7 show possible scenarios of crossover from quantum to thermal regimes. Let us discuss just two of them. The first prescription is following. Fix the temperature $T_{0}$ somewhere in the range $T_{0 b a}>T_{0}>T_{0 a}$. Then align the external field in the $a$ direction and increase its intensity to the point marked "1" on the left diagram in Fig. 7 . The system is in the quantum creep regime now. Then increase the external field beyond $\frac{1}{2} H_{T}$. The system jumps to the point " 2 " of the left diagram and finds itself in the thermal creep regime. Doing the same operations in the reverse order one enforces the system to crossover from thermal to quantum creep regime. The other scenario is the following. Apply magnetic field along $a$ axis and increase its intensity above $\frac{1}{2} H_{T}$ keeping temperature constant in the interval $T_{0 b}>T_{0}>T_{0 a}$. Then move the direction of the external field from $a$ to $b$ axis. The system goes now from point " 2 " of the left diagram (thermal creep) to the point " 3 " of the right diagram (quantum creep). 


\section{Antiferromagnetic superconductors \\ ACKNOWLEDGMENTS}

Illuminating discussions with P. Tekiel, K. Rogacki and M. Ciszek are gratefully acknowledged. This research is supported by the State Committee for Scientific Research (KBN) within the Project No. 2 P03B 12519.

\section{REFERENCES}

1. For review see Superconductivity in Ternary Compounds, edited by M. B. Maple, and $\varnothing$. Fischer, Springer-Verlag, Berlin, 1982.

2. J. Kasperczyk and P. Tekiel Acta Phys. Polon. A 57, 11 (1980)

3. L. N. Bulaevskii, A. I. Buzdin, M. Kulić and S. V. Panjukov, Advances in Physics 34, 176 (1985); Sov. Phys. Uspekhi 27, 927(1984).

4. M. B. Maple, Physica B 215, 110(1995) .

5. L. Bauernfeind, W. Widder and H. F. Braun, Physica C 254, 151(1995) .

6. D. J. Pringle, J. L. Tallon, B. G. Walker and H. J. Trodahl, Phys. Rev. B 59, R11679 (1999).

7. P. W. Klamut, B. Dabrowski, S. Kolesnik, M. Maxwell and J. Mais, Phys. Rev. B 63, 224512 (2001).

8. M. Houzet, A. I. Buzdin and M. Kulić, Phys. Rev. B 64, 184501(2001).

9. S. S. Saxena, P. Agarwal, K. Ahilan, F. M. Grosche, R. K. W. Haselwimmer, M. J. Steiner, E. Pugh, I. R. Walker, S. R. Julian, P. Monthoux, G. G. Lonzarich, A. Huxley, I. Sheikin, D. Braithwaite and J. Flouquet, Nature 406, 587(2000).

10. C. Pfleiderer, M. Uhlarz, S. M. Hayden, R. Vollmer, H. v.Lohneysen, N. R. Bernhoeft and G. G. Lonzarich, Nature 412, 58(2001).

11. N. K. Sato, N. Aso, K. Miyake, R. Shiina, P. Thalmeier, G. Varelogiannis, C. Geibel, F. Steglich, P. Fulde and T. Komatsubara, Nature 410, 340(2001).

12. T. Krzysztoń, J. Mag. Mag. Mater. 15-18, 1572(1980).

13. T. Krzysztoń, Phys. Letters A 104, 225(1984).

14. H. Iwasaki, M. Ikebe and Y. Muto, Phys. Rev. B 33, 4669 (1986).

15. A. I. Buzdin, S. S. Krotov and D. A. Kuptsov, Solid State Commun. 75, $229(1990)$.

16. O. Wong, H, Umezawa and J. P. Whitehead, Physica C158, 32(1989).

17. K. Rogacki, E. Tjukanoff and S. Jaakkola, Phys. Rev. B 64, 094520(2001).

18. W. Thomlinson, G. Shirane, D. E. Moncton, M. Ishikawa and Ø. Fischer, J. Appl. Phys. 50, 1981(1979).

19. W. Thomlinson, G. Shirane, J. W. Lynn and D. E. Moncton, in Superconductivity in Ternary Compounds, edited by M. B. Maple, and Ø. Fischer, SpringerVerlag, Berlin 1982.

20. M. Tinkham, Introduction to Superconductivity, chapter 5, McGraw-Hill Inc., New York 1975.

21. T. Krzysztoń and K. Rogacki to be published.

22. J. W. Lynn, J. Alloys and Compounds 181, 419(1992).

23. J. Zaretsky, C. Stassis, A. I. Goldman, P. C. Canfield, P. Dervenagas, B. K. Cho and D. C. Johnston, Phys. Rev. B 51, 678(1995). 


\section{T. Krzysztoń}

24. J. W.Lynn, T. W. Clinton, W-H. Li, R. W. Erwin, J. Z .Lin, R. N. Shelton and P.Klavins, J. Appl. Phys. 67, 4533(1990).

25. For review see K-H. Müller and V. N. Narozhnyi, Rep. Prog. Phys. 64, 943(2001).

26. S. K. Sinha, J. W. Lynn, T. E. Grigereit, Z. Hossain, L. C. Gupta, R. Nagarajan and C. Godard, Phys. Rev. B 51, 681(1995).

27. R. Szymczak, M. Baran, L. Gładczuk, H. Szymczak, Z. Drzazga and A. Winiarska, Physica C 254, 124(1995).

28. M. R. Eskildsen, A. B. Abrahamsen, D. Lopez, P. L. Gammel, D. J. Bishop, N. H Andersen, K. Mortensen and P. C. Canfield, Phys. Rev. Lett. 86, 320(2001).

29. P. C. Canfield, P. L. Gammel and D. J. Bishop Physics Today 10, 40(1998).

30. T. Krzysztoń, Phys.Lett. A 190 (1994) 196.

31. J. R. Clem and M. W. Coffey, Phys. Rev. B 42, 6209(1990).

32. V. G. Kogan, Phys. Lett. A 85, 298(1981).

33. J. R. Clem, in Proceedings of the 13th Conference on Low Temperature Physics (LT 13), vol. 3, Plenum-Press, New York 1974, p. 102.

34. S. Chakravarty, B. I. Ivlev and Y. N. Ovchinnikov, Phys. Rev.B 42, 2143(1990).

35. E. H. Brandt, Physica C 195, 1(1992).

36. T. Krzysztoń, Physica $C$ 294, 47(1998).

37. P. Tekiel, Z. Phys. B 104, 423(1997).

38. G. Blatter, M. V. Feigelman, V. B. Geshkenbein and A. I. Larkin, Rev. Mod. Phys. 66, 1125(1994).

39. B. I. Ivlev, Yu. N. Ovchinnikov and R. S. Thompson, Phys. Rev. B 44, 7023(1991).

40. W. M. Gaber and B. N. Achar, Phys. Rev. B 52, 1314(1995),

41. T. Krzysztoń, Physica C 340, 156(2000).

42. A. O. Caldeira and A. J. Leggett, Ann.Phys. (N.Y) 149, 374(1983).

43. L. D. Landau and E. M. Lifshitz, Quantum Mechanics, Oxford, Pergamon Press 1962

44. H. Suhl, Phys. Rev. Lett. 14, 226(1965). 